\begin{tabular}{|c|l|}
\hline Title & Nucleophilic A ddition of A Ikanenitriles to A Idehy des via N-Silyl Ketene Imines Generated In Situ \\
\hline Author(s) & Tanino, Keiji; Y oshimura, Fumihiko; Saito, Hiroki; A be, Taiki \\
\hline Citation & $\begin{array}{l}\text { Synlett, 28(14), 1816-1820 } \\
\text { https://doi.org/10.1055/s0036-1588424 }\end{array}$ \\
\hline Issue Date & 2017-09 \\
\hline Doc URL & http://hdl.handle.net/2115/71394 \\
\hline Type & article (author version) \\
\hline Note & Supporting information for this article is avail lable online at https://doi.org/10.1055/s 0036-1588424. \\
\hline File Information & Synlett28_14_1816-1820.pdf \\
\hline
\end{tabular}

Instructions for use 


\section{Nucleophilic Addition of Alkanenitriles to Aldehydes via N-Silyl Ketene Imines Generated In Situ}

\author{
Fumihiko Yoshimura*a \\ Hiroki Saito ${ }^{b}$ \\ Taiki Abe \\ Keiji Tanino*a \\ a Department of Chemistry, Faculty of Science, Hokkaido \\ University, Sapporo 060-0810, Japan \\ ${ }^{b}$ Department of Chemistry, School of Science, Hokkaido \\ University, Sapporo 060-0810, Japan \\ ' Graduate School of Chemical Sciences and Engineering, \\ Hokkaido University, Sapporo 060-0810, Japan \\ fumi@sci.hokudai.ac.jp \\ ktanino@sci.hokudai.ac.jp
}

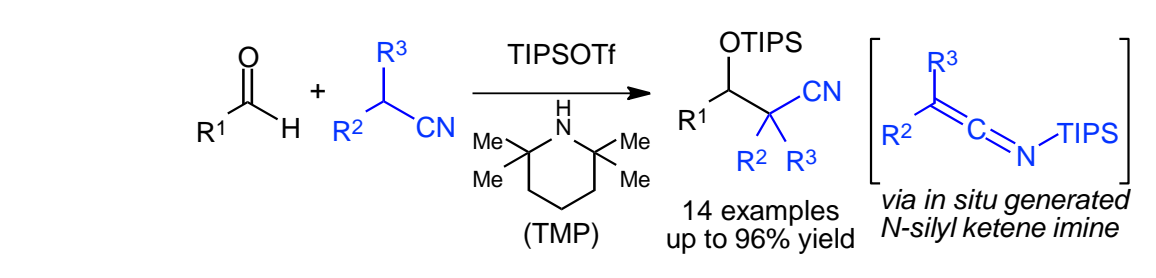

(TMP) up to $96 \%$ yield

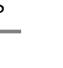

ktanino@sci.hokudai.ac.jp

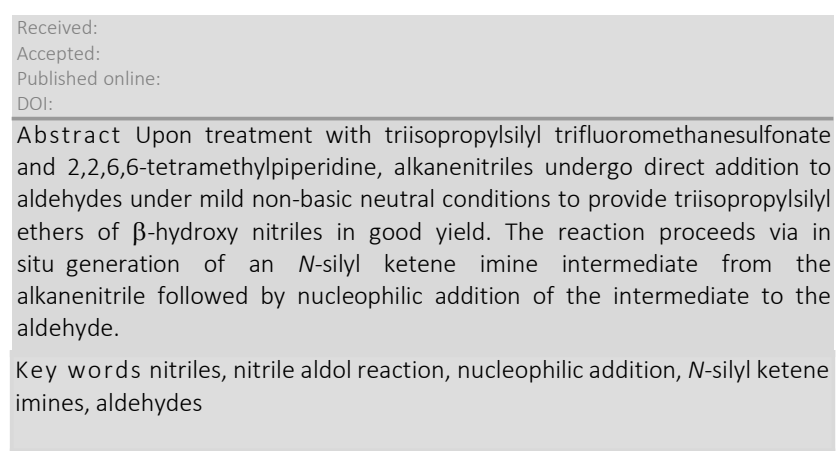

Nucleophilic addition of nitriles to carbonyl compounds (i.e., the nitrile aldol reaction) is a useful transformation in organic synthesis because of the high synthetic utility of $\beta$ hydroxynitrile products owing to the versatile convertibility of the nitrile functionality.1,2 Traditionally, the reaction is carried out through deprotonation of nitriles followed by addition to carbonyl compounds. ${ }^{3}$ However, the generation of $\alpha$-cyano carbanions from simple unactivated alkanenitriles (e.g., $\mathrm{p} K_{\mathrm{a}} 31.3$ in dimethyl sulfoxide for acetonitrile) ${ }^{4}$ requires a stoichiometric amount of a strong base such as lithium diisopropylamide (LDA), which is incompatible with base-sensitive substrates. In addition, strongly basic conditions sometimes cause undesirable reactions, including $\beta$-elimination by dehydration to give the corresponding $\alpha, \beta$-unsaturated nitriles and retro-additions. Recently, several types of catalytic activation of nitriles as nucleophiles have been established, which has led to successful metal-catalyzed nucleophilic additions of unactivated alkanenitriles to aldehydes. ${ }^{5}$ However, these catalytic reactions still have some drawbacks, for which excess amounts of nitrile ( $>5$ equiv) are generally required. Thus, a new methodology that allows the nucleophilic addition of unactivated alkanenitriles to carbonyl compounds under mild reaction conditions without requiring excess substrates would be highly valuable.

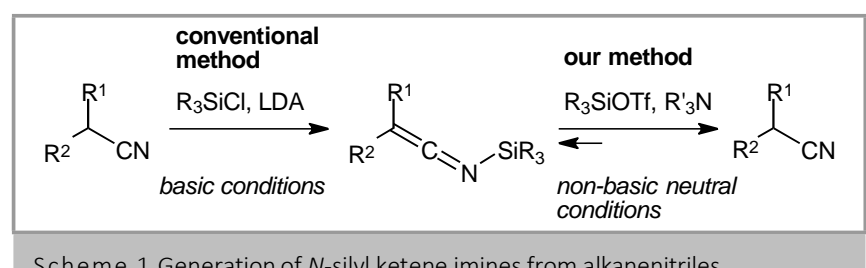

Scheme 1 Generation of $N$-silyl ketene imines from alkanenitriles

$N$-Silyl ketene imines, which are typically prepared by lithiation of alkanenitriles with a strong base such as LDA followed by trapping with a bulky trialkylsilyl chloride, have recently attracted much attention as a competent $\alpha$-cyano carbanion equivalent (Scheme 1).6 Although $N$-silyl ketene imines show synthetic potential, considerable drawbacks remain in their handling and storage instability because they are rapidly hydrolyzed with water. In this context, we reported that $\mathrm{N}$-silyl ketene imines could be generated in equilibrium by treatment of alkanenitriles with trialkylsilyl triflate $\left(\mathrm{R}_{3} \mathrm{SiOTf}\right)$ and a tertiary amine (Scheme 1).7,8 These mild and non-basic generation conditions allowed the development of several C-C bond forming reactions that do not require isolation of the labile $\mathrm{N}$ silyl ketene imines. ${ }^{7}$

Motivated by our interest in new reaction development by using in situ generated $\mathrm{N}$-silyl ketene imines, we expected that treatment of a mixture of aldehydes $\mathbf{1}$ and alkanenitriles $\mathbf{2}$ with $\mathrm{R}_{3}$ SiOTf and tertiary amine would directly yield $O$-trialkylsilyl $\beta$ hydroxy nitriles 3 (Scheme 2).9,10 The reaction would proceed through in situ formation of the highly electrophilic silyl oxonium intermediate $\mathbf{4}$ and $\mathrm{N}$-silyl ketene imine $\mathbf{5}$, followed by addition of $\mathbf{5}$ to $\mathbf{4}$. We report herein trialkylsilyl triflate and alkylamine promoted novel nucleophilic addition reactions of nitriles to aldehydes, which offer an efficient synthetic method for $\beta$-hydroxy nitrile derivatives under non-basic mild conditions. This new method does not require preformation of the labile $N$-silyl ketene imine nucleophile or excess substrates. This is the nitrile analogue of a formal one-pot Mukaiyama aldol reaction. 


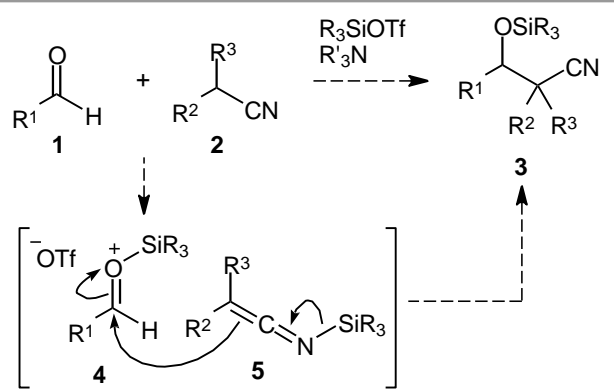

Scheme 2 Our reaction design for nucleophilic addition of alkanenitriles to aldehydes

To ascertain the feasibility of the proposed addition reaction, we initially tested the reaction of benzaldehyde (6) with phenylacetonitrile (7; Scheme 3). When 6 (1 equiv) and 7 (1 equiv) were treated with triethylsilyl trifluoromethanesulfonate (TESOTf; 2 equiv) and triethylamine (2 equiv) in 1,2dichloroethane (DCE), the expected $O$-TES $\beta$-hydroxynitrile 8 was obtained in $41 \%$ yield as a $51: 49$ diastereomeric mixture; however, a considerable amount $(52 \%$ yield $)$ of the corresponding desilylated product $\mathbf{9}$ was also obtained due to the instability of the TES ether in the reaction medium. To suppress this desilylation product, we next tested the ability of a more robust silyl group in the reaction. Happily, the use of triisopropylsilyl trifluoromethanesulfonate (TIPSOTf) led to quantitative formation of $O$-TIPS $\beta$-hydroxynitrile 10. However, the reaction of $\mathbf{6}$ with the sterically hindered nitrile $\mathbf{1 1}$ gave inferior results with the TIPSOTf/ $\mathrm{Et}_{3} \mathrm{~N}$ system, and addition product 12 was obtained in 64\% yield along with TIPS ether $\mathbf{1 3}$ ( $7 \%$ yield), which is derived from hydrosilylation of $\mathbf{6}$ as a side reaction product. 11

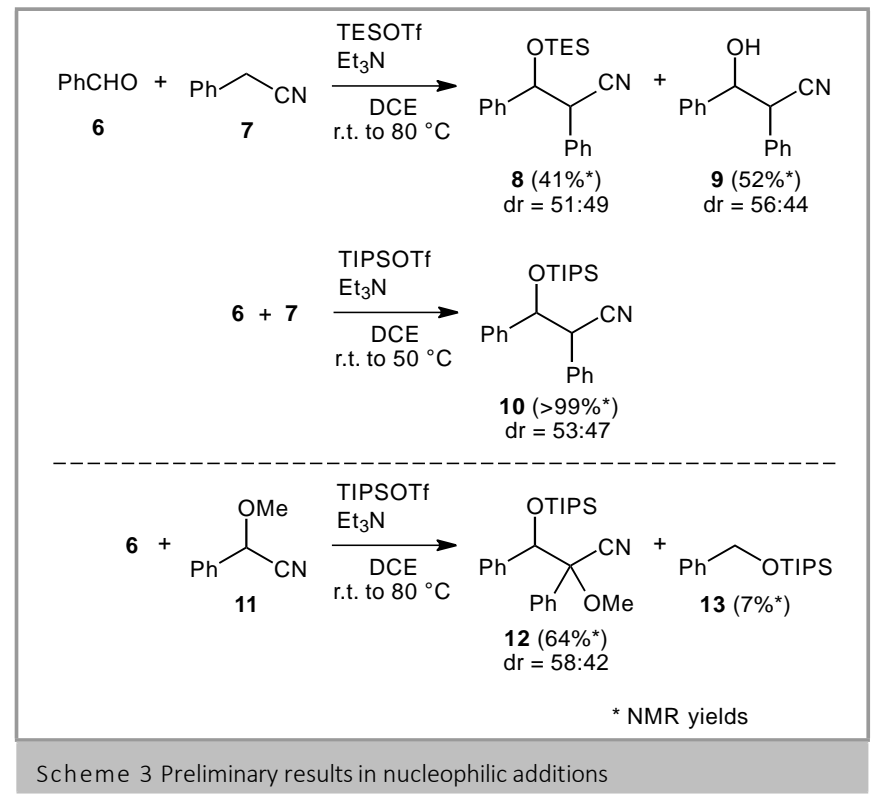

To prevent the competitive hydrosilylation reaction, the effects of amine bases were evaluated in the reaction of nitrile $\mathbf{1 1}$ with benzaldehyde (6; Table 1), which revealed that the choice of amine was of critical importance for this type of reaction. In contrast to the results with triethylamine, significant amounts of TIPS ether $\mathbf{1 3}$ were obtained when sterically hindered tertiary alkylamines, such as diisopropylethylamine (DIPEA) and 1,2,2,6,6-pentamethylpiperidine (PMP), ${ }^{12,13}$ were used (entries 1 and 2). Less hindered bicyclic tertiary amines, such as 1,4diazabicyclo[2.2.2] octane (DABCO) and 2,6-lutidine, did not give addition product 12 , even if the mixture was heated to $80{ }^{\circ} \mathrm{C}$. To our delight, the best result was obtained when 2,2,6,6tetramethylpiperidine (TMP) was used as the amine base, which provided 12 in $85 \%$ yield after isolation ( $d r=55: 45)$ with stirring at room temperature for $22 \mathrm{~h}$ (entry 5). Other sterically hindered secondary amines, such as hexamethyldisilazane (HMDS), did not promote the nucleophilic addition (entry 6). Control experiments revealed that the reaction did not proceed in the absence of either TIPSOTf or TMP. In addition, reduction in the amounts of TIPSOTf and TMP to 1.1 equivalents each resulted in the formation of 12 in only $47 \%$ yield ( $d r=56: 44)$, as well as recovery of $\mathbf{1 1}$ in $22 \%$ yield, after stirring the reaction mixture at room temperature for $23 \mathrm{~h}$. Therefore, the use of two equivalents of both reagents is important for full conversion in this reaction. When TMP was used as the amine base, other silyl triflates, such as trimethylsilyl trifluoromethanesulfonate, TESOTf, and tert-butyldimethylsilyl trifluoromethanesulfonate, also induced nucleophilic additions of hindered nitriles (see Scheme S1 in the supporting information for details). Unfortunately, although the reaction proceeds with a high yield, diastereoselectivity was not induced, probably due to a low level of stereodiscrimination in the addition step.

Table 1 Optimization of Amines for Addition of Nitrile 11 to Benzaldehyde

$(6)^{\mathrm{a}}$

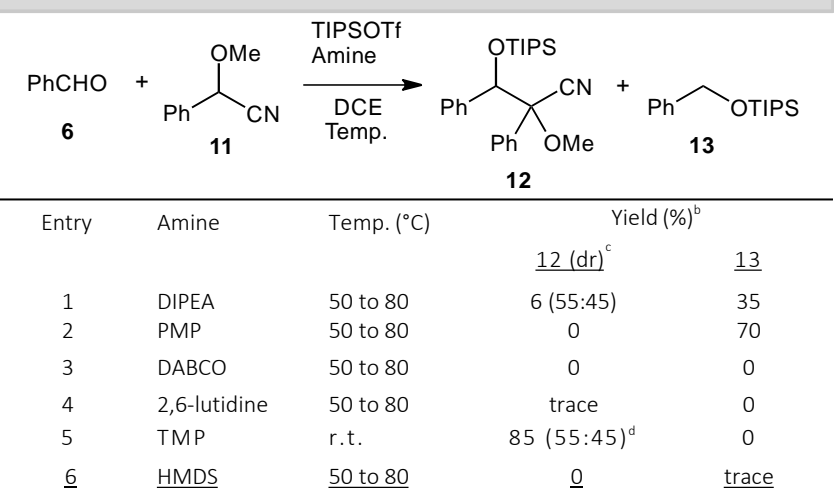

a Reaction conditions: aldehyde $6(0.4 \mathrm{mmol})$, nitrile 11 (0.4 mmol), TIPSOTf $(0.8$ $\mathrm{mmol})$, amine (0.8 mmol), DCE ( $2 \mathrm{~mL}), 80 \mathrm{~min}-22 \mathrm{~h}$.

${ }^{b}$ Yield determined by ${ }^{1} \mathrm{H}$-NMR spectroscopic analysis of the crude product mixture by using pyrazine as an internal standard.

${ }^{c}$ Diastereomeric ratios of 12 are given in parentheses. The relative configuration was not assigned.

${ }^{d}$ Yield of isolated product after purification by silica gel column chromatography.

With the optimized reaction conditions using the TIPSOTf/TMP system in hand, we next investigated the reactions of benzaldehyde (6) with a series of nitriles (Table 2). The results show that the reaction has broad applicability to nitriles including simple unactivated alkanenitriles (i.e., 14a, 14b, and 14e). The $\alpha$-alkyl nitriles 14a,b, $\alpha$-aryl nitriles 7 and 14c,d, acetonitrile (14e), and $\alpha$-halo nitrile $\mathbf{1 4 f}$ afforded the corresponding $O$-TIPS $\beta$-hydroxy nitriles in good to excellent yields (entries 1-7). Note that the reactions of unactivated nitriles 14a and 14b with 6 under the TIPSOTf/Et ${ }_{3} \mathrm{~N}$ system provided no addition products $\mathbf{1 5 a}, \mathbf{b}$, which clearly indicated the unique and remarkable reactivity of TMP as an amine base. ${ }^{14}$ $\alpha, \alpha$-Disubstituted nitriles including isobutyronitrile (14b), 2phenylpropanenitrile (14c), and diphenylacetonitrile (14d) 
gave addition products with a newly formed quaternary carbon atom in good yields, indicating the advantage of the sterically unhindered nitrile group (entries 3-5). When an equimolar amount of acetonitrile was used in this addition, an inseparable mixture of the desired product 15e, the double aldol type addition product (not shown), and the $C$-silylation product, i.e., 2-(triisopropylsilyl)acetonitrile, was obtained. Thus, excess amounts of nitrile were used in the case of acetonitrile (14e) to prevent these side reactions (entry 6). Although chloroacetonitrile (14f) produced the desired product in good yield (entry 7), ${ }^{15}$ fluoroacetonitrile $(\mathbf{1 4 g})$ remained unreactive (entry 8). The diastereomeric ratio of addition products $\mathbf{1 5}$, however, remained low (68:32-50:50).

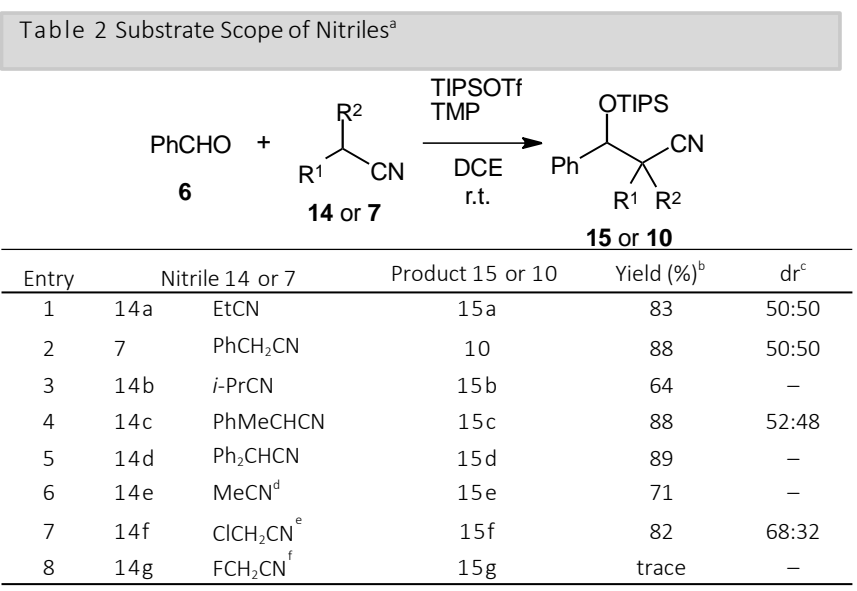

${ }^{a}$ Reaction conditions: aldehyde $6(0.4 \mathrm{mmol})$, nitrile 14 or $7(0.4 \mathrm{mmol})$, TIPSOTf (0.8 mmol), TMP $(0.8 \mathrm{mmol})$, DCE $(2.0 \mathrm{~mL})$, r.t., $30 \mathrm{~min}-140 \mathrm{~min}$.

${ }^{b}$ Yield of isolated product after purification by silica gel column chromatography. ${ }^{c}$ Determined by ${ }^{1} \mathrm{H}-\mathrm{NMR}$ spectroscopic analysis of the crude product; relative stereochemistry not assigned.

${ }^{d}$ Acetonitrile was used as the solvent (0.2 M). Two equivalents of TIPSOTf and TMP were used.

e Toluene was used as the solvent. Reaction temperature was $100^{\circ} \mathrm{C}$

${ }^{\mathrm{f}}$ Reaction temperature was $80^{\circ} \mathrm{C}$.

The synthetic advantage of our new method over the standard method under anionic conditions ${ }^{3}$ is clearly demonstrated in Scheme 4. Namely, our addition reaction of base-sensitive 3(benzyloxy)propanenitrile (14h) to benzaldehyde (6) with TIPSOTf and TMP gave addition product $\mathbf{1 5 h}$ in $81 \%$ yield. By contrast, $\beta$-hydroxy nitrile $\mathbf{1 6}$ was not obtained under the conventional anionic conditions with LDA as a base because competitive $\beta$-elimination of the benzyloxy group from the resulting $\alpha$-cyano carbanion occurred even at $-78^{\circ} \mathrm{C}$.

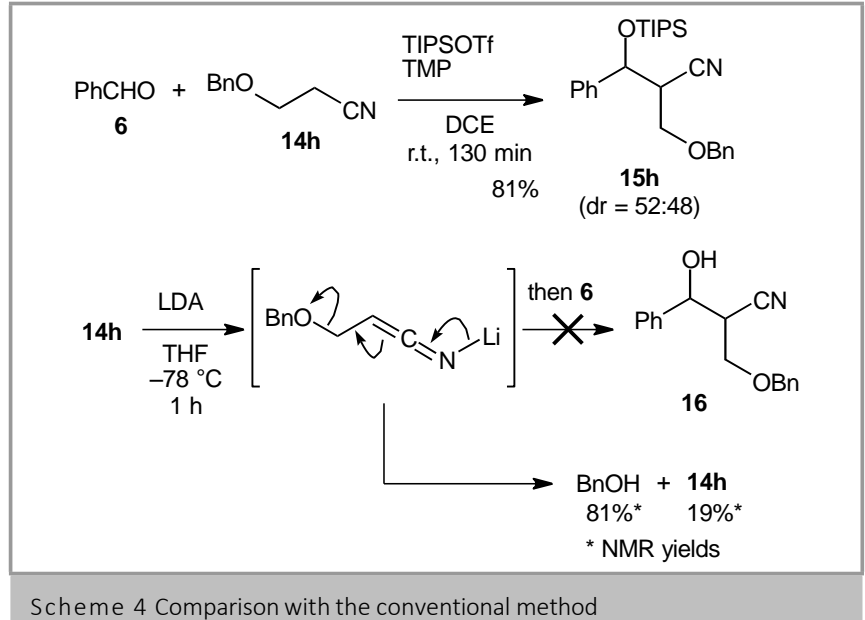

The scope of applicable aldehydes was then examined with phenylacetonitrile (7; Table 3). Addition of nitrile $\mathbf{7}$ to aromatic aldehydes $\mathbf{1 7 a - c}$ with either electron-donating or -withdrawing substituents proceeded smoothly to afford $O$-TIPS $\beta$-hydroxy nitriles 18a-c in high yields (entries 1-3). An ester functionality survived intact under these conditions (entry 3 ). The reaction of sterically hindered 2,6-dimethylbenzaldehyde (17d) efficiently provided addition product 18d (entry 4). The new addition reaction was also applicable to an aliphatic aldehyde without an acidic $\alpha$-proton (entry 5). However, the reaction with enolizable aliphatic aldehyde $\mathbf{1 7 f}$ gave addition product $\mathbf{1 8 f}$ as a minor product (35\% yield) along with silyl enol ether 19, which is derived from 17f (51\% yield; entry 6 and Figure 1). $\alpha, \beta$ Unsaturated aldehyde $\mathbf{1 7 g}$ underwent both 1,2 - and 1,4addition, with 1,4-addition favored, to produce a mixture of TIPS ether 18g (18\% yield, entry 7), silyl enol ether 20 ( $46 \%$ yield, Figure 1), and aldehyde 21 (22\% yield, Figure 1); this indicates a similar tendency to the uncatalyzed solvent-free reaction of preformed $N$-silyl diphenylketene imine. ${ }^{9}$

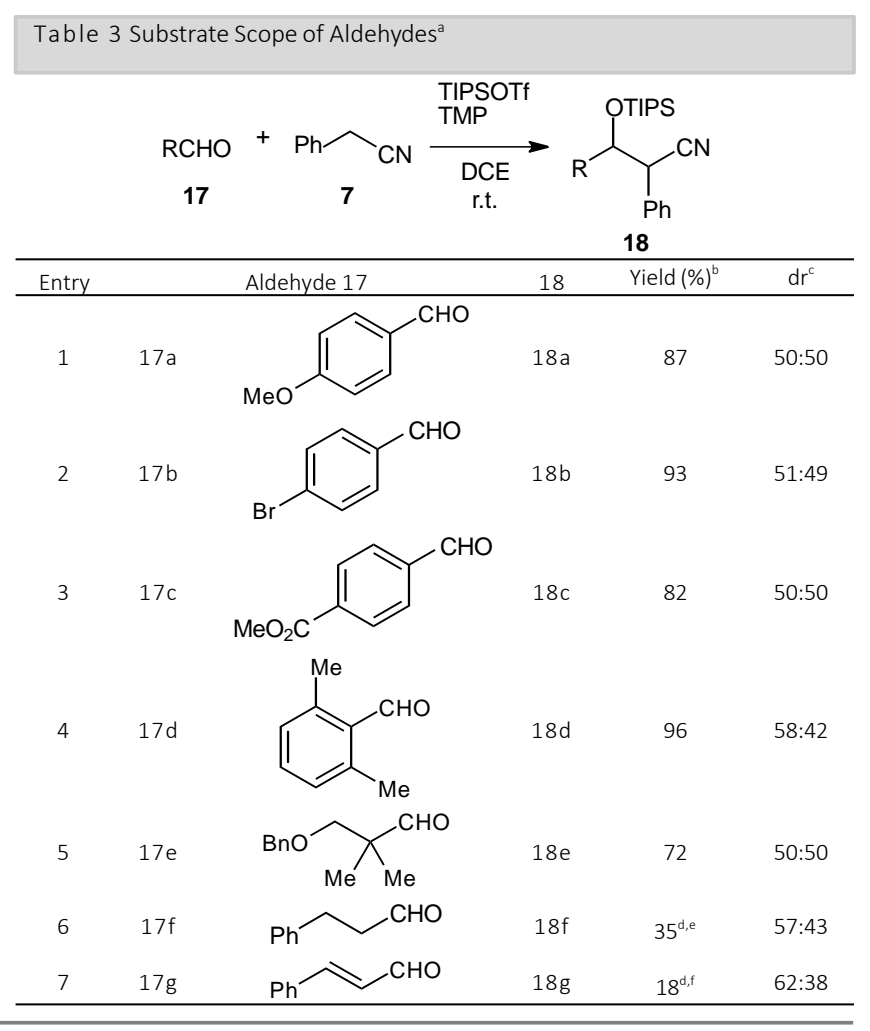


a Reaction conditions: aldehyde 17 (0.4 mmol), nitrile 7 (0.4 mmol), TIPSOTf (0.8 $\mathrm{mmol})$, TMP $(0.8 \mathrm{mmol})$, DCE $(2.0 \mathrm{~mL})$, r.t., $10 \mathrm{~min}$ to $4 \mathrm{~h}$.

${ }^{b}$ Yield of isolated product after purification by silica gel column chromatography. Determined by ${ }^{1} \mathrm{H}$-NMR spectroscopic analysis of the crude product; relative stereochemistry not assigned.

${ }^{\mathrm{d}}$ For side products, see Figure 1

e Slow and dropwise addition of aldehyde $17 \mathrm{f}$ over $2.5 \mathrm{~h}$ to the reaction mixture did not improve the yield of addition product $18 \mathrm{f}$.

${ }^{\mathrm{f}}$ Two-step yield after treatment of the crude product with silica gel in $\mathrm{CH} \mathrm{Cl}_{2}{ }_{2}$

\begin{tabular}{|l|cc|}
\hline & OTIPS & OTIPS \\
$19(51 \%$ Yield $)$ & $20(46 \%$ Yield $)$ & $21(22 \%$ Yield $)$ \\
$E / Z=14: 86$ & $\mathrm{dr}=59: 41$ & $\mathrm{dr}=54: 46$ \\
\hline Figure 1 Side products for entries 6 and 7 in Table 3
\end{tabular}

Finally, nucleophilic addition to a ketone was briefly examined to evaluate the synthetic potential of this reaction (Scheme 5). Thus, upon treatment with TIPSOTf and TMP at room temperature, nitrile 7 underwent an efficient addition reaction with benzophenone (22) to afford adduct $\mathbf{2 3}$ in $97 \%$ yield.

\begin{tabular}{|c|c|}
\hline$\underset{\substack{22 \\
\text { (1 equiv) }}}{\mathrm{O}_{\mathrm{Ph}}}+\overbrace{\mathrm{Ph}_{(1 \text { equiv })}^{7}}^{\overbrace{\mathrm{CN}}}$ & 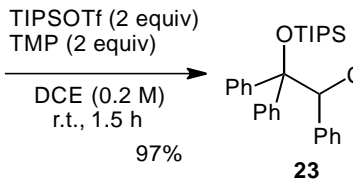 \\
\hline
\end{tabular}

In conclusion, we have developed a novel method for nucleophilic addition reactions of alkanenitriles, including simple unactivated nitriles, to aldehydes promoted by TIPSOTf and TMP under mild silylation conditions. ${ }^{16}$ The reaction appears to proceed via in situ $\mathrm{N}$-silyl ketene imine formation followed by a Mukaiyama aldol-type reaction. ${ }^{17}$ The synthetic benefits of the reaction include the avoidance of preformation and isolation of labile $\mathrm{N}$-silyl ketene imines. The non-basic mild reaction conditions mean that the present method tolerates many functional groups and provides $\beta$-hydroxy nitrile products with a high yield without $\beta$-elimination and retro-additions, which sometimes occur with conventional anionic conditions. The new method does not require excess substrates and will offer an efficient route to $\beta$-hydroxy nitrile derivatives, which serve as useful intermediates in the synthesis of natural products and biologically active substrates. Further studies will focus on the reaction of in situ generated $N$-silyl ketene imines to other classes of electrophiles.

\section{Acknowledgment}

We acknowledge Dr. Eri Fukushi and Mr. Yusuke Takata (GC-MS \& NMR Laboratory, Faculty of Agriculture, Hokkaido University) for performing mass spectral measurements. This work was supported by a Grant for Basic Science Research Projects from The Sumitomo Foundation (to F.Y.) and JSPS KAKENHI Grant Numbers JP15K01795 (to F.Y.), JP15H03806 (to K.T.), and JP15H05842 in Middle Molecular Strategy (to K.T.).

\section{References and Notes}

(1) For reviews of nitrile-containing natural products and pharmaceuticals, see: (a) Fleming, F. F. Nat. Prod. Rep. 1999, 16, 597. (b) Fleming, F. F.; Yao, L.; Ravikumar, P. C.; Funk, L.; Shook, B. C. J. Med. Chem. 2010, 53, 7902.

(2) For utilization in the synthesis of biologically active substrates, see: (a) Kamal, A.; Khanna, G. B. G.; Ramu, R. Tetrahedron: Asymmetry, 2002, 13, 2039. (b) Ankati, H.; Zhu, D.; Yang, Y.; Biehl, E. R.; Hua, L. J. Org. Chem. 2009, 74, 1658.

(3) For a review, see: (a) Arseniyadis, S.; Kyler, K. S.; Watt, D. S. Org. React. 1984, 31, 1. For a non-basic nucleophilic addition, see (b) Hamana, H.; Sugasawa, T. Chem. Lett. 1982, 1401.

(4) Bordwell, F. G. Acc. Chem. Res. 1988, 21, 456.

(5) (a) Suto, Y.; Kumagai, N.; Matsunaga, S.; Kanai, M.; Shibasaki, M. Org. Lett. 2003, 5, 3147. (b) Kumagai, N.; Matsunaga, S.; Shibasaki, M. J. Am. Chem. Soc. 2004, 126, 13632. (c) Fan, L.; Ozerov, O. V. Chem. Commun. 2005, 4450. (d) Goto, A.; Endo, K.; Ukai, Y.; Irle, S.; Saito, S. Chem. Commun. 2008, 2212. (e) Chakraborty, S.; Patel, Y. J.; Krause, J. A.; Guan, H. Angew. Chem. Int. Ed. 2013, 52, 7523. (f) Sureshkumar, D.; Ganesh, V.; Kumagai, N.; Shibasaki, M. Chem. Eur. J. 2014, 20, 15723.

(6) For a review of $N$-silyl ketene imines, see: (a) Denmark, S. E.; Wilson, T. W. Angew. Chem. Int. Ed. 2012, 51, 9980. For recent examples, see: (b) Nishimoto, Y.; Nishimura, T.; Yasuda, M. Chem. Eur. J. 2015, 21, 18301. (c) Sasaki, M.; Ando, M.; Kawahata, M.; Yamaguchi, K.; Takeda, K. Org. Lett. 2016, 18, 1598.

(7) (a) Yoshimura, F.; Torizuka, M.; Mori, G.; Tanino, K. Synlett 2012, 23, 251. (b) Yoshimura, F.; Abe, T.; Tanino, K. Synlett 2014, 25, 1863. (c) Yoshimura, F.; Abe, T.; Tanino, K. Org. Lett. 2016, 18, 1630.

(8) Emde, H.; Simchen, G. Synthesis 1977, 636.

(9) It has been reported that $\mathrm{N}$-(trimethylsilyl)diphenylketene imine undergoes nucleophilic addition to several benzaldehyde derivatives under solvent-free conditions; see: Cazeau, P.; Llonch, J.-P.; Simonin-Dabescat, F.; Frainnet, E. J. Organomet. Chem. 1976, $105,145$.

(10) For, Lewis base catalyzed nucleophilic addition of $N$-silyl ketene imines to aldehydes, see: Denmark, S. E.; Wilson, T. W.; Burk, M. T.; Heemstra, J. R. J. Am. Chem. Soc. 2007, 129, 14864.

(11) For related hydrosilylation reactions mediated by a silyl triflate and a tertiary alkylamine, see: (a) Downey, C. D.; Fleisher, A. S.; Rague, J. T.; Safran, C. L.; Venable, M. E.; Pike, R. D. Tetrahedron Lett. 2011, 52, 4756. (b) Ho, C.; Chan, C.; He, L. Angew. Chem. Int. Ed. 2015, 54, 4512 .

(12) The combination of TIPSOTf and PMP smoothly promoted hydrosilylation of benzaldehyde (6) to afford TIPS ether 13 in $82 \%$ yield (Scheme 6 ).

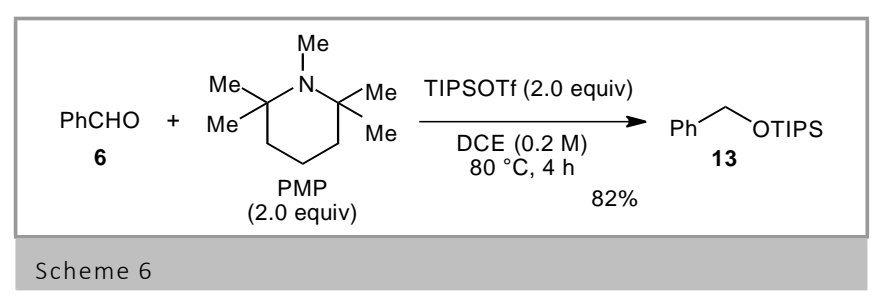

(13) A deuterium-labeling experiment proved that PMP acts as the hydride source (Scheme 7).

\section{Supporting Information}

YES

\section{Primary Data}

NO 


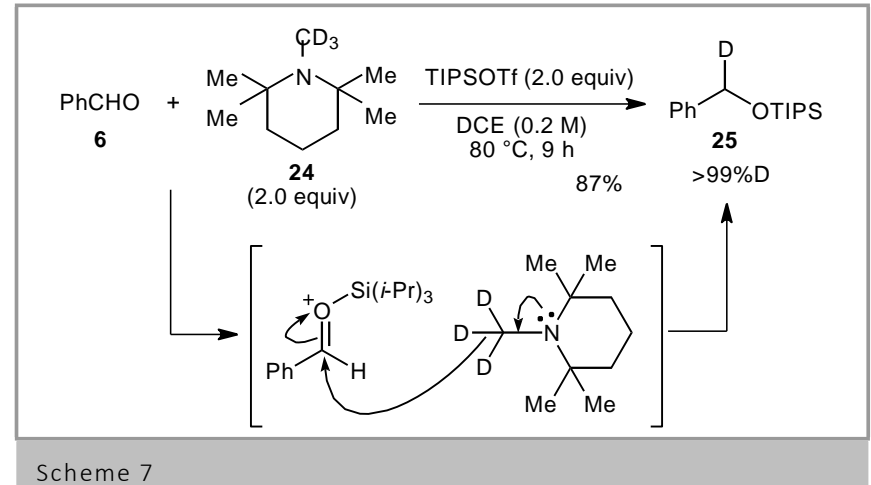

(14) The reason for the superior reactivity of TMP is not clear yet. Very low solubility of 2,2,6,6-tetramethylpiperidinium triflate (TfOH-TMP) in DCE might cause the equilibrium shifts slightly toward the $\mathrm{N}$-silyl ketene imine.

(15) When DCE was used as the solvent, a significant amount of inseparable double aldol type addition product accompanied $\mathbf{1 5 f}$. Solvent screening revealed that toluene could suppress such side reactions, although it required heating of the reaction mixture to $100^{\circ} \mathrm{C}$.

(16) General Procedure (Table 1, Entry 5): To a mixture of benzaldehyde (6; $40.8 \mu \mathrm{L}, \quad 0.400 \quad \mathrm{mmol}), \quad$ 2-methoxy-2phenylacetonitrile $(\mathbf{1 1} ; 55.5 \mu \mathrm{L}, 0.400 \mathrm{mmol})$, and 2,2,6,6tetramethylpiperidine $(136 \mu \mathrm{L}, 0.800 \mathrm{mmol})$ in DCE $(2.0 \mathrm{~mL})$ was added TIPSOTf (215 $\mu \mathrm{L}, 0.800 \mathrm{mmol}$ ), and the mixture was stirred at room temperature for $22 \mathrm{~h}$, at which point the consumption of starting materials $\mathbf{6}$ and $\mathbf{1 1}$ was complete (as determined by TLC analysis, hexane:EtOAc $=4: 1$ ). After cooling to $0{ }^{\circ} \mathrm{C}$, the reaction was quenched by slow addition of saturated aqueous $\mathrm{NaHCO}_{3}(1 \mathrm{~mL})$, and the resulting mixture was filtered through a cotton plug to remove the precipitate (rinsed with $\mathrm{CH}_{2} \mathrm{Cl}_{2}$ ). The filtrate was extracted with $\mathrm{CH}_{2} \mathrm{Cl}_{2}(1 \mathrm{~mL} \times 3)$. The combined organic extracts were dried over $\mathrm{MgSO}_{4}$ and concentrated under reduced pressure. The residue was purified by flash column chromatography $\left(\mathrm{SiO}_{2}\right.$, hexane:EtOAc $\left.=50: 1\right)$ to give nitrile 12 (139.6 mg, $0.341 \mathrm{mmol}, 85 \%$ yield) as an inseparable 55:45 mixture of diastereomers.

Compound 12: Colorless oil; ${ }^{1} \mathrm{H}-\mathrm{NMR}\left(500 \mathrm{MHz}, \mathrm{CDCl}_{3}\right) \delta$ 7.53-7.51 (1H, m), 7.40-7.36 (3H, m), 7.31-7.27 (2H, m), 7.22 $(1 \mathrm{H}, \mathrm{t}, J=7.4 \mathrm{~Hz}), 7.13-7.04(2 \mathrm{H}, \mathrm{m}), 6.92(1 \mathrm{H}, \mathrm{m}), 4.98(0.55 \mathrm{H}, \mathrm{s})$, $4.97(0.45 \mathrm{H}, \mathrm{s}), 3.34(0.45 \times 3 \mathrm{H}, \mathrm{s}), 3.18(0.55 \times 3 \mathrm{H}, \mathrm{s}), 1.15-1.09$ $(0.45 \times 3 \mathrm{H}, \mathrm{m}), 1.06(0.45 \times 9 \mathrm{H}, \mathrm{d}, J=6.9 \mathrm{~Hz}), 1.00(0.45 \times 9 \mathrm{H}, \mathrm{d}, J=$ $7.5 \mathrm{~Hz}), 0.81-0.74(0.55 \times 21 \mathrm{H}, \mathrm{m}) ;{ }^{13} \mathrm{C} \mathrm{NMR}\left(125 \mathrm{MHz}, \mathrm{CDCl}_{3}\right) \delta$ 139.08, 137.95, 134.79, 133.93, 129.21, 128.99, 128.51, 128.45, 128.18, 128.09, 127.96, 127.86, 127.76, 127.41, 127.20, 127.07, $117.15,116.89,88.12,86.64,81.44,81.06,54.03,53.97,17.87$, $17.82,17.70,17.63,12.44,12.40$; IR (ATR) v 2943, 2867, 2365, 1122, $1069 \mathrm{~cm}^{-1}$; HRMS (FD) calcd for $\mathrm{C}_{25} \mathrm{H}_{36} \mathrm{NO}_{2} \mathrm{Si}\left([\mathrm{M}+\mathrm{H}]^{+}\right)$: 410.2515, found: 410.2484 .

(17) Attempts to detect $N$-silyl ketene imine intermediates by ${ }^{1} \mathrm{H}$ - or ${ }^{13} \mathrm{C}$-NMR were unsuccessful, suggesting that these reactive species in equilibration with the corresponding nitriles are existing only in low concentration. The reaction of $\mathbf{6}$ with $\mathbf{1 1}$ did not proceed in the absence of either TIPSOTf or TMP. The alkanenitrile underwent isomerization at the $\alpha$-position of the cyano group by treatment with TIPSOTf/TMP (i.e., nitrile $\mathbf{2 8}$ in Scheme S2). These results support that the nucleophilic addition should proceed via the $\mathrm{N}$-silyl ketene imine intermediate. For details, see Scheme S2 in the Supporting Information. 